\title{
Motion Planning in Environments with Low Obstacle Density*
}

\author{
A. F. van der Stappen, M. H. Overmars, M. de Berg, and J. Vleugels \\ Department of Computer Science, Utrecht University, \\ P.O. Box 80.089, 3508 TB Utrecht, The Netherlands \\ \{frankst, markov, markdb, jules\}@cs.uu.nl
}

\begin{abstract}
We present a simple and efficient paradigm for computing the exact solution of the motion planning problem in environments with a low obstacle density. Such environments frequently occur in practical instances of the motion planning problem. The complexity of the free space for such environments is known to be linear in the number of obstacles. Our paradigm is a new cell decomposition approach to motion planning and exploits properties that follow from the low density of the obstacles in the robot's workspace. These properties allow us to decompose the workspace, subject to some constraints, rather than to decompose the higher-dimensional free configuration space directly. A sequence of uniform steps transforms the workspace decomposition into a free space decomposition of asymptotically the same size. The approach leads to nearly optimal $O(n \log n)$ motion planning algorithms for free-flying robots with any fixed number of degrees of freedom in workspaces with low obstacle density.
\end{abstract}

\section{Introduction}

An ultimate goal in the field of robotics is the development of robots that accept high-level descriptions of tasks and execute these tasks without intervention from their environment. A fundamental task for such an autonomous robot would be to move from its current free placement to some other specified free placement while avoiding collision with the obstacles on its way. The problem of finding such a collision-free path is referred to as the motion planning problem. Even though most of today's operational robots are not fully autonomous, most of them have to deal with certain instances of the motion planning problem during their operation. The methods that are used in practice to tackle these instances have the notable drawback that they may fail to find an existing path (or spend

\footnotetext{
* This research was supported by the Dutch Organization for Scientific Research (N.W.O.) and by the ESPRIT III BRA Project 6546 (PROMotion).
} 
a lot of time and storage to find one). A direction of research in computational geometry, initiated by a series of papers — known as the piano movers' series [22]-[25], [28] — by Schwartz and Sharir in the early '80s, studies the exact solution of the motion planning problem. Exact methods for solving the motion planning problem are guaranteed to find a path if one exists, and report failure if no path exists. The disadvantage of exact methods is their high worst-case running time. The high worst-case time bounds prevent exact methods from becoming popular alternatives for the solution of practical instances of the motion planning problem. We show, however, that certain realistic assumptions on the robot and its environment allow for a simple general approach to the solution of exact motion planning problems. The approach leads to several very efficient motion planning algorithms for such instances.

We focus on the following general version of the basic motion planning problem.

Given a robot $\mathcal{B}$ in a workspace $\mathrm{W}$ with a collection $\mathcal{E}$ of closed connected stationary obstacles, and two free placements $Z_{0}$ and $Z_{1}$, find a motion for the robot from $Z_{0}$ to $Z_{1}$ during which it avoids collision with the obstacles, or report that no such motion exists.

The robot $\mathcal{B}$ is assumed to be a collection of closed rigid bodies of total constant complexity and to have $q$ degrees of freedom (DOF). The robot moves in a workspace $\mathrm{W}$, which usually equals the Euclidean space of dimension two $\left(\mathbb{R}^{2}\right)$ or three $\left(\mathbb{R}^{3}\right)$. The motion of the robot is constrained by a set $\mathcal{E}$ of $n$ pairwise disjoint obstacles. Each obstacle $E \in \mathcal{E}$ is a closed connected constant-complexity subset of the workspace W. The obstacles do not change place or shape.

The motion planning problem is commonly modeled and solved in the configuration space $C$, which is the space of parametric representations of robot placements. The dimension of $C$ equals the number of degrees of freedom $q$ of the robot $\mathcal{B}$. A point $Z \in C$ (representing a robot placement) is referred to as a configuration. Although there is a subtle difference between a placement and a configuration, we use both terms interchangeably. The free space $\mathrm{FP}$ is the subspace of $C$ consisting of points that represent placements of the robot in which it does not intersect any obstacle in $\mathcal{E}$ :

$$
\mathrm{FP}=\left\{Z \in C \mid \mathcal{B}[Z] \cap\left(\bigcup_{E \in \mathcal{E}} E\right)=\emptyset\right\},
$$

where $\mathcal{B}[Z]$ stands for the set of workspace points covered by $\mathcal{B}$ in configuration $Z$. The free space can be regarded as the union of certain cells - the free cells-in the arrangement $^{1}$ of constraint hypersurfaces. A constraint hypersurface is the set of placements in which a robot feature, i.e., a basic part of the boundary like a vertex, edge, or face, touches an obstacle feature of appropriate dimension. The constraint hypersurfaces are assumed to be algebraic and to have bounded degree. A collision-free path or motion for a robot $\mathcal{B}$ from an initial placement $Z_{0}$ to a final placement $Z_{1}$ is a continuous map: $\tau:[0,1] \rightarrow \mathrm{FP}$, with $\tau(0)=Z_{0}$ and $\tau(1)=Z_{1}$. Hence, solving the motion planning

\footnotetext{
${ }^{1}$ The arrangement of a set is the subdivision of space into connected pieces of any dimension induced by that set.
} 
problem boils down to finding a continuous curve in FP connecting $Z_{0}$ and $Z_{1}$. The effort that is required to find such a curve clearly depends on the complexity of the free space.

Exact motion planning algorithms process the free space into a query structure that allows for the efficient solution of one or more path-finding queries. Although there essentially exist two different approaches to exact motion planning (cell decomposition and retraction), the time spent in processing the free space and the size of the resulting query structure clearly depend on the complexity of the free space. Cell decomposition algorithms (see, e.g., [11], [15], [22]-[25], and [28]) partition the free space into a finite number of simple connected subcells, such that planning a motion between two placements in a single subcell is straightforward and such that uniform crossing rules can be defined for $\mathcal{B}$ crossing from one cell into another. Each cell defines a vertex in the connectivity graph $\mathrm{CG}$. Two vertices in CG are connected by an edge if their corresponding subcells share a common boundary allowing direct crossing of the robot. Given the graph $\mathrm{CG}$, the motion planning problem is reduced to a graph problem: determine a sequence of pairwise connected nodes connecting the nodes corresponding to the subcells containing the initial and final placements of $\mathcal{B}$. The imposed simplicity of the subcells facilitates the transformation of the sequence of subcells into an actual collision-free motion for the robot. The desired simplicity of the subcells in the cell decomposition, however, also causes the number of subcells to depend on the complexity of the free space. As a result, the size of the query structure - the connectivity graph CG-and the time to compute it depend on the complexity of FP. Retraction methods (see, e.g., [7], [13], [17], [18], and [29]) aim at capturing the structure and connectivity of the free space in some one-dimensional network of curves in the free space, the roadmap. The curves are chosen in such a way that a simple collision-free motion connects every point $Z \in \mathrm{FP}$ to some point $\operatorname{Im}(Z)$ on the roadmap, and such that all curves in a single connected component of the free space are connected. Given a roadmap with these properties, the problem of finding a motion between two free placements $Z_{0}$ and $Z_{1}$ is reduced to the problem of finding a sequence of roadmap curves connecting the roadmap points $\operatorname{Im}\left(Z_{0}\right)$ and $\operatorname{Im}\left(Z_{1}\right)$. The desired properties of the roadmap, however, also cause the number of curves to depend on the complexity of the free space. As a result, the size of the query structure - the roadmap - and the time to compute it depend on the complexity of FP.

The complexity of the free space is determined by the number of multiple contacts of the robot $\mathcal{B}$. A multiple contact of the robot $\mathcal{B}$ is a placement in which it touches more than one obstacle feature. Besides the collisions of the robot with the obstacles, parts of the robot can also collide with other robot parts. Although these so-called self-collisions are often ignored in our considerations, we return to them later to demonstrate the validity of the results when self-collisions are taken into account. Unfortunately, the number of multiple contacts, and, hence, the complexity of the free space, can be very high. Under our circumstances where the total number of obstacle features is $\Theta(n)$ and the number of features of the $q$-DOF robot is bounded by a constant, the free space complexity can be $\Omega\left(n^{q}\right)$. As a generic example, consider the robot arm in Fig. 1. If the square obstacles are sufficiently small and, within each column, sufficiently close together, then the number of $q$-fold contacts is easily seen to be $\Omega\left(n^{q}\right)$. As a consequence, the complexity of the free space for the robot arm is $\Omega\left(n^{q}\right)$. Slightly lower worst-case free space complexities have been obtained for specific free-flying rigid robots (like convex polyhedra) among certain classes of obstacles (like polyhedra). These bounds generally remain close to 


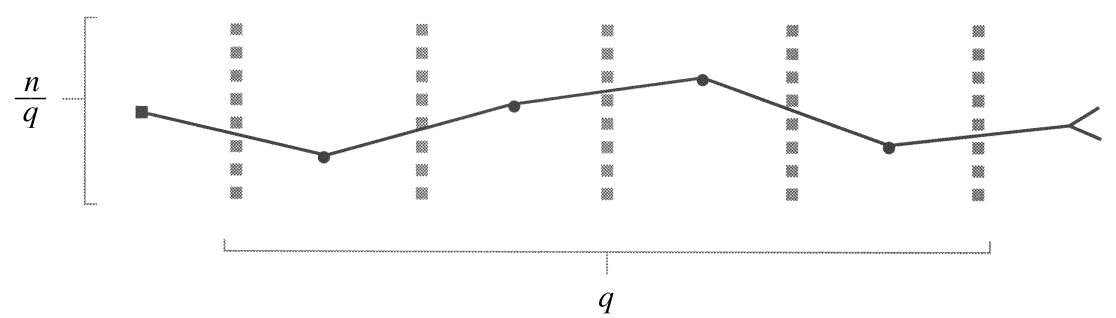

Fig. 1. A ( $q$-DOF) robot arm consisting of $q$ links with $\Omega\left(n^{q}\right) q$-fold contacts, and, hence, with free space complexity $\Omega\left(n^{q}\right)$.

one order of magnitude, i.e., a factor $n$, below the $\Omega\left(n^{q}\right)$ bound (see, e.g., [14] and [27]). Hence, even in such more specific cases, the theoretical worst-case bounds are high. Fortunately, in many practical situations the complexity of the free space is much smaller, as artificially constructed workspaces with, e.g., a very large robot and many small obstacles, are not encountered very often in real life. When extreme shapes and sizes of the robot and the obstacles do not occur, high free space complexities tend to be harder to obtain. Consider for example the motion planning environment of Fig. 2 where the 6-DOF "spider" robot and the obstacles have roughly the same sizes. While being in contact with a certain obstacle, the robot is unable to touch more than a constant number of other obstacles. Then the number of multiple contacts cannot exceed $O(n)$. Hence, the free space for this robot has complexity $O(n)$ and thus remains far below the free space complexity obtained with the construction of Fig. 1. The impressive gap between the $\Omega\left(n^{q}\right)$ construction and the $O(n)$ example immediately raises the question what specific properties of the robot and the obstacles lead to low free space complexities. What natural mild assumptions would, for example, lead to the relative low obstacle density of the above example, in which the robot is unable to touch more than a constant number of obstacles simultaneously?

Van der Stappen et al. [32] show that the combinatorial complexity of the free space is linear in the number of obstacles if the robot is not too large compared with the obstacles and if any workspace region intersects no more than a constant number of obstacles that are at least as large as the region. We refer to the latter property as the low obstacle density property of the workspace. (Actually, in [32] the linear bound is only proven for

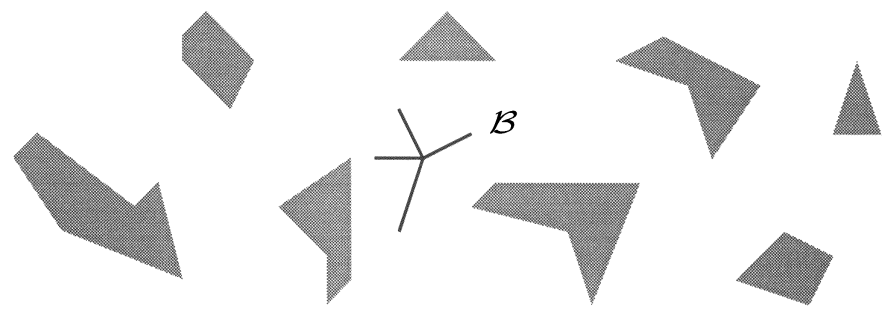

Fig. 2. A 6-DOF robot with few multiple contacts, and, hence, with low free space complexity. 
the more restricted assumption of fatness of the obstacles. It is though trivial to extend it to sets that satisfy the low obstacle density property.) Circumstances that resemble the low obstacle density have also been studied by Schwartz and Sharir [26] who refer to it as bounded local complexity and by Pignon [21] who calls it sparsity.

A question that immediately comes to mind when considering the combinatorial result of [32] is whether this reduced complexity opens the way to efficient motion planning algorithms for such realistic environments. The vast majority of motion planning algorithms have no reported sensitivity to the complexity of the free space. A clear exception is the boundary-vertices retraction algorithm by Sifrony and Sharir [29] for a ladder moving in a planar workspace with polygonal obstacles. The algorithm runs in time $O(K \log n)$, where $K$ is the number of pairs of obstacle corners that lie less than the length of the ladder apart. The low obstacle density causes $K$ to be only $O(n)$, whereas it could be $\Theta\left(n^{2}\right)$ in the worst case for arbitrary workspaces with obstacles. Some algorithms have a hidden sensitivity to the complexity of the free space [31]. For example, the boundary cell decomposition algorithm by Avnaim et al. [3], running in time $O\left(n^{3} \log n\right)$ for a constant complexity polygonal robot amidst arbitrary polygonal obstacles, can be shown to run in $O(n \log n)$ time in the low obstacle density setting. The $O\left(n^{5}\right)$ algorithm by Schwartz and Sharir [22] for planning the motion of a ladder or a polygonal robot amidst polygonal obstacles can be shown to run, unmodified, in time $O\left(n^{2}\right)$ if the obstacle density is low, whereas a minor modification improves the efficiency to a running time of $O(n \log n)$ (see also [30]). Hence, there exist (planar) motion planning algorithms that do benefit from low free space complexities, even though several other algorithms do not. Algorithms for efficient motion planning in three-dimensional workspaces are scarce: approaches in contact space, like the algorithms mentioned above by Sifrony and Sharir, and by Avnaim et al., were never shown to generalize to higher dimensions. General approaches to motion planning (e.g., by Schwartz and Sharir [23] with running time $O\left(n^{2^{q+6}}\right)$ and Canny [7] with running time $\left.O\left(n^{q} \log n\right)\right)$ are computationally expensive, even under our beneficial circumstances. These rigorous methods do not take advantage of the structure that is present in the free space in our case.

In this paper we present a new paradigm for motion planning in environments with low obstacle density. The idea is that we do not compute a decomposition of the free configuration space but of the workspace. Next, we "lift" this workspace decomposition into the configuration space. We show that the low obstacle density guarantees that this lifting can be done without increasing the asymptotic complexity of the decomposition. The realistic low obstacle density and bounded robot size assumptions also guarantee the existence of a workspace partition of (optimal) $O(n)$ size, based on the binary space partition by de Berg [4]. The computation of the partition takes $O(n \log n)$ time. As a result, motion planning problems in low obstacle density environments can be solved in $O(n \log n)$ time. The dependence on the number $q$ of degrees of freedom of the robot is restricted to the hidden constants.

We are aware of only few (related) results on exact motion planning methods with provable efficiency or free space complexity-sensitive behavior for realistic motion planning problems (with low complexity workspaces or free spaces). The running time of Sifrony and Sharir's algorithm [29] depends on the number of pairs of obstacle corners that lie less than the length of the ladder apart. This number gives some idea of how cluttered the obstacles in the workspace are and is closely related to the complexity of 
the free space. Schwartz and Sharir [26] consider workspaces with obstacles of so-called bounded local complexity. A set of obstacles has bounded local complexity if the number of obstacles intersecting any ball is bounded by some constant depending on the radius of the ball. Our notion of low obstacle density differs from bounded local complexity in that it only imposes a constant bound on the number of larger obstacles intersecting any region. The authors give directions on how to solve the motion planning problem among obstacles with bounded local complexity. Pignon [21] processes two-dimensional workspaces with polygonal obstacles and a polygonal robot (using Minkowski differences) to detect and solve simple path-finding queries efficiently. Simple queries are queries that are either easily seen to yield no solution-because there exists no path for a simple inscribed shape of the robot—or are easily solvable-because there exists a path for an outer approximation of the robot with fewer degrees of freedom. Alt et al. [2] introduce the tightness of a motion planning problem for a rectangle among polygonal obstacles as a measure for its complexity. The tightness of a problem is closely related to the scaling factor for the rectangular robot to make a solvable problem unsolvable, or an unsolvable problem solvable. The authors present an approximate motion planning algorithm for the rectangular robot with a tightness-dependent running time.

This paper is organized as follows. Section 2 formalizes the notion of a low object density space and shows its relation to fatness. It reports some properties of low object density spaces that, though interesting in their own right, mainly serve as a tool in the subsequent sections. In Section 3 we exploit the low obstacle density property of the workspace to obtain a paradigm for planning the motion of a robot that is not too large compared with the obstacles. The running time of an algorithm based on the paradigm depends on the time to compute some constrained partition of the workspace. Section 4 tackles the problem of finding a small and efficiently computable partition. Section 5 concludes the paper.

\section{Low Obstacle Density and Its Relation to Fatness}

In many practical situations, the complexity of the free space tends to remain far below the theoretical worst-case complexity bounds. Lower complexities particularly occur when the obstacles in the workspace are not cluttered too much and the robot is not too large compared with the obstacles. A clear but very restrictive example of such an environment is a workspace in which the robot can never touch more than one obstacle at a time. Our aim is to find a weaker and more realistic assumption that still leads to a low free space complexity and efficient motion planning algorithms.

\subsection{Low Obstacle Density}

This subsection is devoted to identifying a weak assumption on the workspace and the obstacles so that efficient motion planning is possible. The results are basically reformulations of results previously reported in [32], but we repeat them because they are fundamental to this paper.

As the relative sizes of the robot and the obstacles play a crucial role throughout 
the paper, we first give convenient measures for the size of an obstacle and a robot. We find the radius of the minimal enclosing hypersphere of an obstacle or region the most convenient among the many ways to express the obstacle or region size. For the sake of brevity, we simply refer to the radius of the minimal enclosing hypersphere of an obstacle or region as the size of that obstacle or region. The radius of the minimal enclosing hypersphere of the robot, however, may vary due to the possibility that the robot may consist of several links. We introduce the reach $\rho_{\mathcal{B}}$ of a robot $\mathcal{B}$ as a means of expressing the size of $\mathcal{B}$. Let $O \in \mathcal{B}$ be a reference point on the robot, and assume that the configuration space $C=\mathrm{W} \times D$, where $\mathrm{W}$ is the $d$-dimensional workspace (where a point $p \in \mathrm{W}$ represents a placement of $O)$ and $D$ is the $(q-d)$-dimensional space of the remaining degrees of freedom.

Definition 2.1 (Reach $\rho_{\mathcal{B}}$ of a Robot $\left.\mathcal{B}\right)$. Let $Z_{\mathrm{W}}$ be some arbitrary position of the reference point $O$ of the robot $\mathcal{B}$. Then the reach $\rho_{\mathcal{B}}$ of the robot $\mathcal{B}$ is defined as

$$
\rho_{\mathcal{B}}=\sup _{Z_{D} \in D} \max _{p \in \mathcal{B}\left[\left(Z_{\mathrm{W}}, Z_{D}\right)\right]} d\left(p, Z_{\mathrm{W}}\right)
$$

where $d\left(p, Z_{\mathrm{W}}\right)$ denotes the Euclidean distance between the points $p$ and $Z_{\mathrm{W}}$.

In words, the reach $\rho_{\mathcal{B}}$ of a robot $\mathcal{B}$ is the maximum distance in the workspace that any point in the robot $\mathcal{B}$ can ever have to the reference point. Notice the natural similarity of the measures of sizes of the robot and the obstacles: the reach of the robot is its maximum size, taken over all its possible placements.

The definition in the previous paragraph allows us to impose an explicit bound on the ratio of the sizes of the robot and the obstacles. This bound is one of the two keys to a low free space complexity and to efficient motion planning algorithms. Assuming that the size of each obstacle in the workspace is at least $\rho$, the restriction we impose is that the reach $\rho_{\mathcal{B}}$ of the robot $\mathcal{B}$ is bounded by $b \cdot \rho$, for some constant $b \geq 0$. Definition 2.2 (see also [34]) defines a class of (work)spaces that, in combination with the bound on the relative size of the robot and the obstacles, give rise to a linear complexity free space and allow for efficient motion planning.

Definition 2.2 (Low (Object/Obstacle) Density). Let $\mathcal{E}$ be a set of objects in $\mathbb{R}^{d}$. We say that $\mathbb{R}^{d}$ with $\mathcal{E}$ has low (object) density if any region of size $\sigma$ intersects at most a constant number of objects in $\mathcal{E}$ of size at least $\sigma$.

In the specific case that $\mathbb{R}^{d}$ is the workspace $\mathrm{W}$ of a robot, and $\mathcal{E}$ is the set of obstacles in $\mathrm{W}$, we refer to $\mathrm{W}$ as a low obstacle density workspace.

Lemma 2.3 follows easily if we realize that we can cover a region of size $c \cdot \sigma$ by $(\lfloor 2 c\rfloor+1)^{d}$ hyperspheres of radius $\sigma$, each of which intersects no more than a constant number of objects.

Lemma 2.3. Let $\mathbb{R}^{d}$ with a set $\mathcal{E}$ of objects satisfy the low object density property. Then any region of size $c \cdot \sigma$, for some constant $c \geq 0$, intersects at most a constant number of objects $E \in \mathcal{E}$ of size at least $\sigma$. 
Another immediate consequence of the low object density property is that every point $p \in \mathbb{R}^{d}$ lies in at most a constant number of objects $E \in \mathcal{E}$. This fact, in conjunction with Theorem 2.4 and the invariance of the low density property under moderate inflation of the objects (expressed by Theorem 2.9), is crucial in the considerations of Section 3.

Theorem 2.4. Let $\mathbb{R}^{d}$ with a set $\mathcal{E}$ of $n$ constant-complexity objects satisfy the low object density property. Then the complexity of the arrangement $\mathcal{A}(\partial \mathcal{E})$ of all object boundaries $\partial E$ is $O(n)$.

Proof. We assume that the objects in $\mathcal{E}$ are ordered by increasing size: $E_{1}, \ldots, E_{n}$ and $\rho_{1} \leq \cdots \leq \rho_{n}$, where $\rho_{i}$ is the size of $E_{i}$. We count for each object boundary $\partial E_{i}$ the subspaces of dimensions 0 through $d-1$ that are defined by its intersection with object boundaries $\partial E_{j}$ with $j>i$. A boundary $\partial E_{i}$ can only be intersected by a boundary $\partial E_{j}$ $(j>i)$ if the minimal enclosing hypersphere $S_{i}$ (with radius $\rho_{i}$ ) of $E_{i}$ is intersected by $E_{j}$. Definition 2.2 yields that there can only be a constant number of such $E_{j}$ 's, so there is at most a constant number of boundaries $\partial E_{j}(j>i)$ that intersect $\partial E_{i}$. By the additional assumption that all objects and thus their boundaries have constant complexity, there is only a constant number of constant-complexity subspaces of dimension between 0 and $d-1$ defined by the intersection of $\partial E_{i}$ and boundaries $\partial E_{j}(j>i)$. Adding the contributions of all $n$ boundaries amounts to a total of $O(n)$ subspaces of dimensions 0 to $d-1$ in the arrangement $\mathcal{A}(\partial \mathcal{E})$. The linear bounds on the number of these subspaces imply the same bound of $O(n)$ on the number of $d$-faces in $\mathcal{A}(\partial \mathcal{E})$, making the total combinatorial complexity of the arrangement $O(n)$.

Theorem 2.5 states the linear complexity result for the free space for motion planning problems in low density environments. The reader is referred to [32] for a proof.

Theorem 2.5. Let $\mathrm{W}$ with a set $\mathcal{E}$ of $n$ constant-complexity obstacles of size at least $\rho$ be a low obstacle density workspace. The free space for a constant-complexity robot $\mathcal{B}$ with reach $\rho_{\mathcal{B}} \leq b \cdot \rho$, for some constant $b \geq 0$, moving in $\mathrm{W}$ has complexity $O(n)$.

In the next subsection we consider an interesting class of motion planning environments that satisfy the low obstacle density property. An immediate consequence of the preceding results will be that complexity of the free space is linear in the number of obstacles.

\subsection{Fatness}

Fatness has turned out to be an interesting phenomenon in computational geometry. Several papers present surprising improvements in combinatorial complexity bounds [2], [8], [12], [16], [32] and efficiency gains for algorithms [1], [4], [10], [19], [20] if the objects under consideration are fat. Fat objects are "compact" to some extent, rather than long and thin. Fatness is a realistic assumption, since in many practical instances of geometric problems the considered objects are fat. The aim of studying fatness is to 
find new fast and simple algorithms or to demonstrate improved efficiency of existing algorithms for such practical instances. The achievements of the study of fatness so far include near-linear bounds on the complexity of the union of certain fat figures (e.g., triangles, wedges) in the plane [2], [8], [12], [16], a linear bound on the complexity of the free space for motion planning amidst fat obstacles [32], and efficient algorithms for computing depth orders on certain fat objects [1], binary space partitions for sets of fat objects [4], hidden surface removal for fat horizontal triangles [10], and range searching and point location among fat objects [19], [20].

Contrary to many other definitions of fatness in literature [1], [2], [8], [10], [12], [16], the notion introduced in [32], and recaptured below, applies to general objects in arbitrary dimension $d$. The definition involves a parameter $k$, supplying a qualitative measure of the fatness of an object: the smaller the value of $k$, the fatter the object must be.

Definition 2.6 ( $k$-Fatness). Let $E \subseteq \mathbb{R}^{d}$ be an object and let $k$ be a positive constant. The object $E$ is $k$-fat if, for all hyperspheres $S \in U_{E}$,

$$
k \cdot \operatorname{volume}(E \cap S) \geq \operatorname{volume}(S),
$$

where $U_{E}$ consists of all hyperspheres centered inside $E$ and not fully containing $E$.

According to the definition, a $k$-fat object $E$ must cover at least $(1 / k)$ th of any hypersphere that is centered inside $E$ but does not fully contain it. The definition of fatness forbids fat objects to be long and thin, or to have long and thin parts.

Obstacle fatness and low obstacle density are closely related. An intuitive explanation lies in the observation that it is impossible to have a large number of fat obstacles of a certain minimum size intersecting a small region. A more formal proof follows from [32]. Here, we confine ourselves to reporting the result.

Theorem 2.7. A space $\mathbb{R}^{d}$ with nonintersecting $k$-fat objects is a low object density space.

\subsection{Object Wrappings}

This subsection shows that the objects in a scene satisfying the low density property can be expanded by an amount proportional to the size of the smallest object without affecting the low density property. Intuitively, the first objects that are to be intersected by expanding obstacles are neighboring objects. As the density of other objects in the vicinity of each object is low, a considerable expansion of the objects is, again intuitively, necessary to create more than a few intersections of object expansions, and, hence, to increase the density of the scene asymptotically. Below, these informal ideas are made specific by giving bounds on the (allowable) expansion of the objects such that the density of the space with the expanded objects remains $O(n)$. The $\varepsilon$-wrappings that are introduced provide a convenient means of expressing the expansion of an object. Sufficiently tight wrappings play a crucial role in providing the justification that the 
paradigm for motion planning in low obstacle density workspaces presented in Section 3 indeed works.

Definition 2.8 ( $\varepsilon$-Wrapping). Let $E \subseteq \mathbb{R}^{d}$ and let $\varepsilon \in \mathbb{R}^{+}$. Any object $\Delta$ satisfying $E \subseteq \Delta \subseteq\left\{p \in \mathbb{R}^{d} \mid d(p, E) \leq \varepsilon\right\}$ is an $\varepsilon$-wrapping of $E$.

An $\varepsilon$-wrapping of an object $E$ is an enclosing shape of $E$, with the property that the distance from the wrapping to $E$ never exceeds $\varepsilon$.

Theorem 2.9 states the circumstances that preserve the low density property of the space while the objects are expanded.

Theorem 2.9. Let $\mathbb{R}^{d}$ with a set $\mathcal{E}$ of objects satisfy the low object density property, and let $\rho$ be a lower bound on the size of any object in $\mathcal{E}$. Let $b \geq 0$ be a constant and assume that $a(b \cdot \rho)$-wrapping $\Delta(E)$ is given for every object $E \in \mathcal{E}$. Then $\mathbb{R}^{d}$ with the set $\{\Delta(E) \mid E \in \mathcal{E}\}$ of wrappings satisfies the low object density property.

Proof. Let $R \subseteq \mathbb{R}^{d}$ be a region of size $\sigma ; R$ intersects at most a constant number of objects from $\mathcal{E}$ of size at least $\sigma$, because $\mathbb{R}^{d}$ with the objects $\mathcal{E}$ satisfies the low object density property. Let $S$ be the hypersphere with a radius $\sigma+b \rho$ and concentric with $R$ 's minimal enclosing hypersphere. We count the number of wrappings of size at least $\sigma$ that intersect $R$. Let $\Delta(E)$ be such a wrapping. We note that if $\Delta(E)$ intersects $R$, then the object $E$ itself must intersect the interior of the hypersphere $S$ with radius $\sigma+b \rho$. Moreover, we note that the size of $E$ itself is at least $\sigma-b \rho$. Thus, we can bound the number of wrappings of size at least $\sigma$ intersecting $R$ by bounding the number of objects of size at least $\sigma-b \rho$ intersecting $S$. We distinguish two cases. If $\sigma \geq 2 b \rho$, then the radius $\sigma+b \rho$ of $S$ satisfies the inequality $\sigma+b \rho \leq 3(\sigma-b \rho)$. By Lemma 2.3, $S$ intersects at most a constant number of objects of size at least $\sigma-b \rho$. If $\sigma \leq 2 b \rho$, then the radius $\sigma+b \rho$ of $S$ satsifies $\sigma+b \rho \leq 3 b \rho$. By Lemma 2.3, $S$ is intersected by at most a constant number of objects.

Informally, Theorem 2.9 says that the low object density property is preserved if the objects in a low density space are expanded by an amount that is at most proportional to the size of the smallest object in the scene. We use Theorem 2.9 mostly in conjunction with Theorem 2.4; together these theorems imply that the arrangement of expanded obstacle boundaries has linear complexity, given that each expanded obstacle has constant complexity.

Besides applications in motion planning, Theorems 2.4 and 2.9 have interesting implications for certain types of arrangements and complexities of union boundaries of certain geometric figures. The relation between the complexity of an arrangement of wrapping boundaries and the complexity of the boundary of the union of the wrappings becomes clear if one realizes that the faces of the union boundary form a subset of the faces of the arrangement of wrapping boundaries. So, under the circumstances sketched in Theorem 2.4, the boundary of the union of all wrappings has complexity $O(n)$. Theorems 2.4 and 2.9 are, for example, applicable to the molecule model in the paper by Halperin and Overmars [9]. The atoms that constitute a molecule are assumed to satisfy 
the hard sphere model. The hard sphere model describes atoms by spheres and forbids any sphere center to get too close to another sphere center. This property allows us to regard the atoms as wrappings of certain nonintersecting smaller spheres, which are only a bounded amount smaller than the original atoms and can be shown to satisfy the low density property. The construction provides an alternative proof for the linear (in the number of atoms) descriptional complexity of the molecule surface.

\section{A Paradigm for Low Density Motion Planning}

The ultimate aim of this paper is to determine a general approach to planning the motion of a not too large, constant-complexity robot moving in a workspace with a low density of constant-complexity obstacles. It has been noted that the existing planar motion planning algorithms are not easily extendible toward other-in particular spatial-problems. Moreover, the existing general approaches to motion planning (like those by Schwartz and Sharir [23] and Canny [7]) are computationally expensive, even for problems from the special class that we consider here.

A configuration space contains constraint hypersurfaces of the form $f_{\varphi, \Phi}$, consisting of placements of the robot $\mathcal{B}$ in which a robot feature $\varphi$ is in contact with an obstacle feature $\Phi$. We denote the fact that $\xi$ is a feature of some object or object set $X$ by $\xi \in_{f} X$. The arrangement of all constraint hypersurfaces $f_{\varphi, \Phi}\left(\varphi \in_{f} \mathcal{B}, \Phi \in_{f} \mathcal{E}\right)$ divides the higher-dimensional configuration space into free and forbidden cells. Even in the case of low density motion planning, the complexity of a single free cell can be $\Omega(n)$, which illustrates that some additional processing is necessary to facilitate efficient motion planning. Naturally, the structure of a higher-dimensional arrangement like the arrangement of constraint hypersurfaces is difficult to understand, let alone to subdivide the free arrangement cells into simple subcells or to capture their structure in some one-dimensional roadmap. At this point, however, the low obstacle density comes to our help to provide us with a very useful property of a $q$-dimensional configuration space $C$ of the form $C=\mathrm{W} \times D$, where $\mathrm{W}$ is the $d$-dimensional workspace and $D$ is some $(q-d)$-dimensional (rest-)space. (Free-flying rigid robots, for example, fit well in this framework. For a free-flying rigid robot in $\mathrm{W}=\mathbb{R}^{3}, D$ is the space defined by the three rotational degrees of freedom of the robot.) The low obstacle density can be shown to result in a very interesting property of configuration space, namely that

$$
\left|\left\{f_{\varphi, \Phi} \mid \varphi \in_{f} \mathcal{B} \wedge \Phi \in_{f} \mathcal{E} \wedge f_{\varphi, \Phi} \cap(p \times D) \neq \emptyset\right\}\right|=O(1)
$$

for each point $p \in \mathrm{W}$. In words, the $(q-d)$-dimensional subspace $p \times D$, obtained by lifting the workspace point $p$ into configuration space, is intersected by only a constant number of constraint hypersurfaces. An immediate consequence of this result is that these algebraic hypersurfaces of bounded degree define a constant-complexity arrangement in each cross section $p \times D$ of the configuration space $C$.

At a more abstract level, low obstacle density motion planning problems for free-flying robots can be regarded as a subclass of the larger class of motion planning problems with configuration spaces $C=B \times D$ that satisfy

$$
\left|\left\{f_{\varphi, \Phi} \mid \varphi \in_{f} \mathcal{B} \wedge \Phi \in_{f} \mathcal{E} \wedge f_{\varphi, \Phi} \cap(p \times D) \neq \emptyset\right\}\right|=O(1),
$$




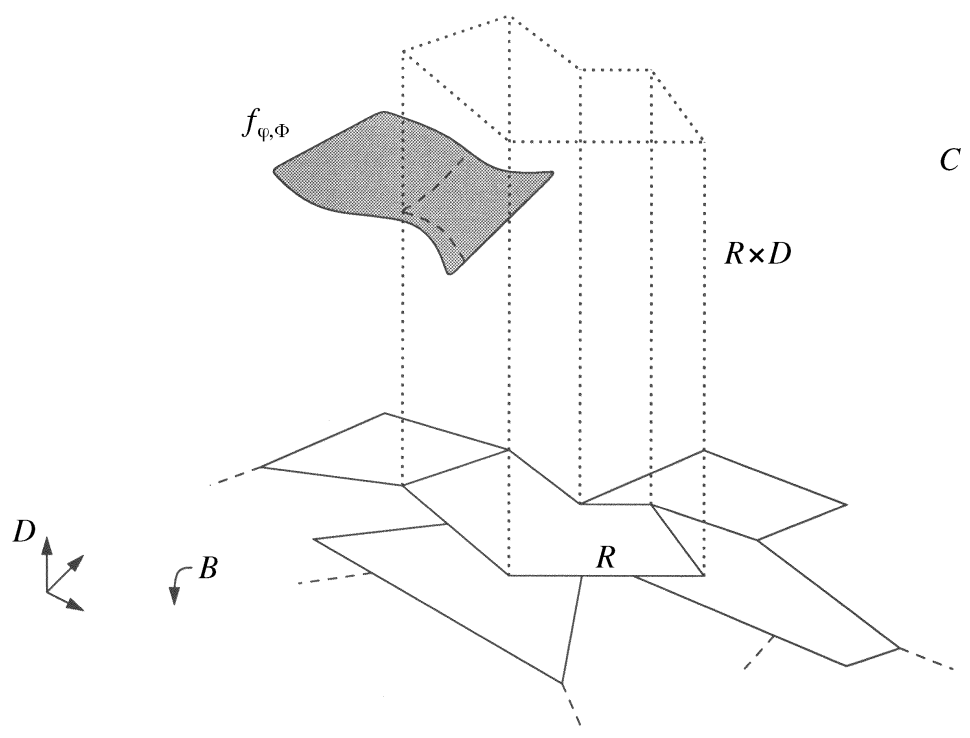

Fig. 3. A three-dimensional example of a cylindrifiable configuration space $C$ with a two-dimensional base space $B$ (and, hence, a one-dimensional rest-space $D$ ), and a fragment of the base partition in $B$. The constraints on the base partition guarantee that the cylinder $R \times D$ is intersected by only a constant number of constraint surfaces $f_{\varphi, \Phi}$.

for each point $p \in B$. A configuration space $C$ that satisfies this constraint will be said to be cylindrifiable. Furthermore, we call the subspace $B$ of $C$ a base space. Hence, low obstacle density motion planning problems for free-flying robots have cylindrifiable configuration spaces in which the workspace constitutes a valid base space. As a result of the cylindrifiability of $C$, it is possible to partition the subspace $B$ into closed regions $R$ (or $C$ into cylinders $R \times D$ ) such that

$$
\left|\left\{f_{\varphi, \Phi} \mid \varphi \in_{f} \mathcal{B} \wedge \Phi \in_{f} \mathcal{E} \wedge f_{\varphi, \Phi} \cap(R \times D) \neq \emptyset\right\}\right|=O(1) .
$$

The partition of $B$ that leads to the cylinders is called the base partition. Figure 3 illustrates the terminology introduced in this paragraph.

We now consider the configuration space cylinder $R \times D$ corresponding to a region $R$ in a base partition in $B$. By the definition of the base partition, the cylinder $R \times D$ is intersected by $O(1)$ constraint hypersurfaces. These algebraic hypersurfaces of bounded degree subdivide $R \times D$ into a constant number of cells. If we furthermore assume that the cylinders themselves have constant descriptional complexity (achievable by establishing that the regions $R$ have constant complexity), then each of the $O(1)$ free or forbidden cells in $R \times D$ has constant complexity as well. In conclusion, the constraint hypersurfaces and the cylinder boundaries divide the free space into constant-complexity subcells, which allow for simple motion planning in their interiors.

The preceding arguments suggest a two-step approach for computing a cell decomposition for a motion planning problem with a cylindrifiable configuration space: first, find a base partition in some appropriate base space $B$ of $C$, and then transform the partition into a cell decomposition of the free space FP $\subseteq C$, by computing a decomposition of 
the free part of every cylinder. We shall see that the resulting decomposition consists of subcells that allow for simple motion planning within their interiors, and that the rules for crossing from one cell into another are simple.

In Section 3.1 it is shown how the latter part of the two-step approach outlined above transforms a base partition into a cell decomposition of comparable size in time proportional to the size of the base partition. Noting this, the problem of finding a (small) cell decomposition of the free space FP $\subseteq C$ reduces to the problem of finding a (small) base partition in an appropriate base space $B \subseteq C$. Section 3.2 exploits specific properties of the constraint hypersurfaces that follow from the shapes and relative positions of the obstacles to simplify the constraints on the partition of the base space $B=\mathrm{W}$ for motion planning problems involving free-flying robots. The new and simpler constraints combined with the transformation steps result in a tailored paradigm for motion planning for robots in environments with low obstacle density. In Section 4 this paradigm is shown to lead to efficient algorithms for motion planning for free-flying robots.

\subsection{Transforming a Base Partition into a Cell Decomposition}

Assume that we are given a graph $\left(V_{B}, E_{B}\right)$, where $V_{B}$ is a set of constant-complexity closed regions $R$ that partition a base space $B$ and individually satisfy

$$
\left|\left\{f_{\varphi, \Phi} \mid \varphi \in_{f} \mathcal{B} \wedge \Phi \in_{f} \mathcal{E} \wedge f_{\varphi, \Phi} \cap(R \times D) \neq \emptyset\right\}\right|=O(1)
$$

and $E_{B}=\left\{\left(R, R^{\prime}\right) \in V_{B} \times V_{B} \mid \partial R \cap \partial R^{\prime} \neq \emptyset\right\}$ contains the adjacencies of $V_{B}$ 's regions. Note that, in this definition, every region is adjacent to itself.

The transformation algorithm given below transforms the graph $\left(V_{B}, E_{B}\right)$ into a connectivity graph $\mathrm{CG}=\left(V_{C}, E_{C}\right)$, consisting of a set $V_{C}$ of constant-complexity subcells that collectively partition the set of free placements FP, and a set $E_{C}=\left\{\left(A, A^{\prime}\right) \in\right.$ $\left.V_{C} \times V_{C} \mid \partial A \cap \partial A^{\prime} \neq \emptyset\right\}$ of subcell adjacencies. The sizes of the sets $V_{C}$ and $E_{C}$ are of the same order of magnitude as the sizes of $V_{B}$ and $E_{B}$, respectively: $\left|V_{C}\right|=O\left(\left|V_{B}\right|\right)$ and $\left|E_{C}\right|=O\left(\left|E_{B}\right|\right)$. Note that the graph $\left(V_{C}, E_{C}\right)$ supports simple path-finding between two placements in subcells $A \in V_{C}$ and $A^{\prime} \in V_{C}$ : the constant complexity of the individual subcells guarantees easy path-finding within a subcell, and the constant complexity of the shared boundary of two adjacent subcells-following from the constant complexity of the involved subcells - caters for simple boundary crossing rules. The transformation steps are independent of the actual motion planning problem under consideration.

$$
\begin{aligned}
& \text { Algorithm TRANSFORM: }\left(V_{B}, E_{B}\right) \longrightarrow\left(V_{C}, E_{C}\right) \\
& V_{C}:=\emptyset ; \\
& E_{C}:=\emptyset ; \\
& \text { for all } R \in V_{B} \text { do }
\end{aligned}
$$

\section{for all $R \in V_{B}$ do}

1. compute the arrangement $\mathcal{A}$ of surfaces $f_{\varphi, \Phi}$ intersecting $R \times D$;

2. use $\mathcal{A}$ to compute a decomposition of $\mathrm{FP} \cap(R \times D)$ into a constant number of constant-complexity subcells $A$;

3. $\operatorname{Desc}(R):=\emptyset$; 


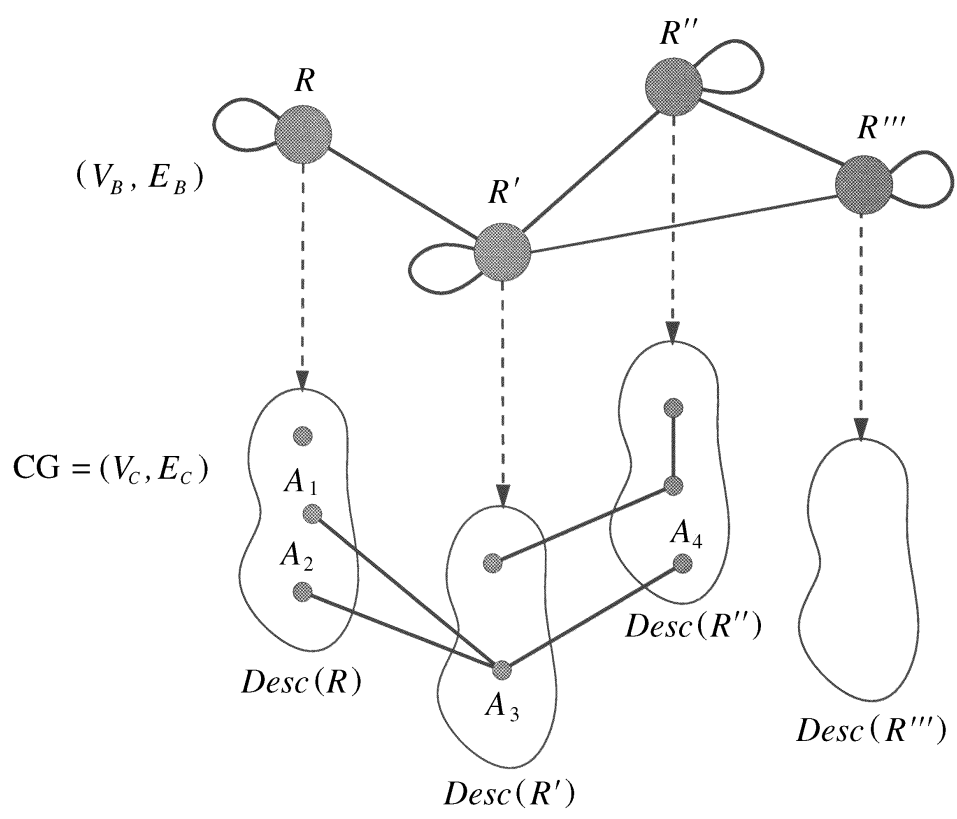

Fig. 4. The relation between the base partition graph $\left(V_{B}, E_{B}\right)$ in the subspace $B$ of $C$ at the top, and the connectivity graph $\mathrm{CG}=\left(V_{C}, E_{C}\right)$ in the configuration space at the bottom. Each node $R \in V_{B}$ defines at most $O(1)$ nodes $A \in V_{C}$, collected in a set $\operatorname{Desc}(R)$. Two nodes $A$ and $A^{\prime}$ in $V_{C}$ can only be connected if the corresponding nodes $R$ and $R^{\prime}$ in $V_{B}$ are connected, so, e.g., $A_{1}$ may be connected to nodes in $\operatorname{Desc}\left(R^{\prime}\right)$ but not to nodes in $\operatorname{Desc}(R)$.

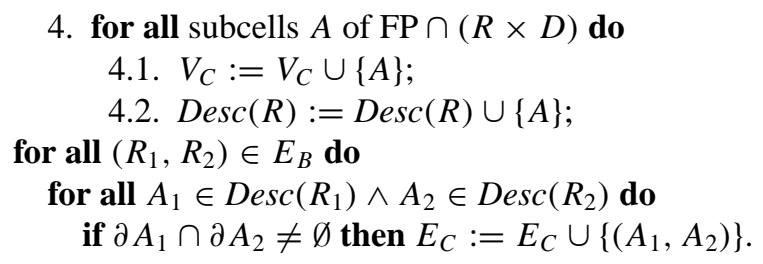

Figure 4 gives a pictorial explanation of the transformation. We review the different steps of the transformation in more detail to verify their validity and to analyze the efficiency. Recall that the definition of the set $V_{B}$ and the constant complexity of the regions $R \in V_{B}$ imply the constant complexity of all subcells $A \in V_{C}$.

The first for-loop computes a decomposition of FP $\cap(R \times D)$ into $O(1)$ (constantcomplexity) subcells and gathers these in a set $V_{C}$. One possible way to perform this computation in constant time is by applying (in step 2) the rigorous techniques by Schwartz and Sharir [23] to the constant number of constraint hypersurfaces intersecting the cylinder $R \times D$. The output is a subdivision of the arrangement cells into constantcomplexity subcells. Restriction of these subcells to $R \times D$ and subsequently filtering out the forbidden ones results in an appropriate cell decomposition of FP $\cap(R \times D)$. Each of the four steps in the loop is easily verified to run in constant time, provided that the constraint hypersurfaces $f_{\varphi, \Phi}$ intersecting $R \times D$ can be determined in constant 
time. If this requirement is indeed satisfied, the entire loop runs in time $O\left(\left|V_{B}\right|\right)$. Upon termination of the first loop, each constant-cardinality set $\operatorname{Desc}(R)$ stores all nodes in $V_{C}$ that correspond to free subcells in $R \times D$.

Two free subcells $A_{1}$ and $A_{2}$ are adjacent if they share a common boundary (which allows for collision-free crossing from one subcell into the other). Such subcells $A_{1}$ and $A_{2}$ can only be adjacent if their containing cylinders $R_{1} \times D \supseteq A_{1}$ and $R_{2} \times D \supseteq A_{2}$ are adjacent in $C$ and, hence, $R_{1}$ and $R_{2}$ are adjacent in $B$. An adjacency $\left(R_{1}, R_{2}\right)$ gives rise to only a constant number of adjacencies of nodes in $A_{1} \in \operatorname{Desc}\left(R_{1}\right)$ and $A_{2} \in \operatorname{Desc}\left(R_{2}\right)$ due to the constant cardinality of $\operatorname{Desc}\left(R_{1}\right)$ and $\operatorname{Desc}\left(R_{2}\right)$. The common boundary of $A_{1}$ and $A_{2}$ has constant complexity because $A_{1}$ and $A_{2}$ have constant complexity. The nested for-loop in the second for-loop takes constant time by the above considerations, implying a running time of $O\left(\left|E_{B}\right|\right)$ for the latter loop. If we combine the time-bounds of the two steps in the transformation algorithm, then we find that the running time depends solely on the size of the base partition in a lower-dimensional subspace of the configuration space.

Lemma 3.1. The algorithm TRANSFORM transforms the graph $\left(V_{B}, E_{B}\right)$ corresponding to a base partition of the base space $B$ into the connectivity graph $\left(V_{C}, E_{C}\right)$ of a cell decomposition of the free space $\mathrm{FP} \subseteq C=B \times D$ in time $O\left(\left|V_{B}\right|+\left|E_{B}\right|\right)$.

Once we have computed the connectivity graph $\left(V_{C}, E_{C}\right)$, the problem of solving a motion planning query "find a free path from a placement $Z_{1}=\left(Z_{1 B}, Z_{1 D}\right)$ to another placement $Z_{2}=\left(Z_{2 B}, Z_{2 D}\right)$ " basically reduces to a point location query with $Z_{1 B}$ and $Z_{2 B}$ in $V_{B}$ to find $R_{1} \ni Z_{1 B}$ and $R_{2} \ni Z_{2 B}$. So, we need a structure for point location in the base space rather than in the full configuration space $C$. After that it takes $O(1)$ time to find $A_{1} \ni Z_{1}$ using $\operatorname{Desc}\left(R_{1}\right)$ and $A_{2} \ni Z_{2}$ using $\operatorname{Desc}\left(R_{2}\right)$, followed by a search in the graph $\left(V_{C}, E_{C}\right)$ for a sequence of subcells connecting $A_{1}$ to $A_{2}$. The constant complexities of the subcells and of the common boundaries of pairs of adjacent subcells facilitate the transformation of the subcell sequence into an actual free path for $\mathcal{B}$.

\subsection{A Tailored Paradigm for Free-Flying Robots}

We now direct our attention to a subset of the class of motion planning problems with cylindrifiable configuration spaces, namely the class of problems involving a not too large constant-complexity robot $\mathcal{B}$ with $q$ degrees of freedom moving in a workspace with constant-complexity obstacles $E \in \mathcal{E}$ that satisfies the property of Definition 2.2, where $q$ is a constant. The restriction on the size of the robot is expressed by a bound on its reach: $\rho_{\mathcal{B}} \leq b \cdot \rho$, where $b$ is some positive constant and $\rho$ is a lower bound on the sizes of the $n$ obstacles in $\mathcal{E}$. For the moment, we assume that the robot $\mathcal{B}$ does not self-collide, that is, no part of $\mathcal{B}$ can collide with any other part of $\mathcal{B}$ during motion. Let $O \in \mathcal{B}$ be a reference point of the robot. The tailored paradigm presented below suits robots with configuration spaces $C=\mathrm{W} \times D$, so that the position of the robot's reference point in the robot's workspace is part of the specification of its placement. A placement $Z$ of the robot can thus be written as $Z=\left(Z_{\mathrm{W}}, Z_{D}\right)$, where $Z_{\mathrm{W}} \in \mathrm{W}=\mathbb{R}^{d}$ and $Z_{D} \in D$. Free-flying robots fit naturally in this framework. 
The robot with its reference point fixed at $p$ can only touch obstacles within a distance $\rho_{\mathcal{B}}$ from the point $p$. Such obstacles clearly intersect the hypersphere with radius $\rho_{\mathcal{B}}$ centered at $p$. Lemma 2.3 says that the number of obstacles of size at least $\rho$ intersecting any region with diameter $2 \rho_{\mathcal{B}} \leq 2 b \cdot \rho$ is bounded by a constant. As all obstacles in $\mathcal{E}$ have size at least $\rho$, the robot $\mathcal{B}$ can touch no more than $O(1)$ obstacles while its reference point remains fixed at $p$. This fact leads to the following lemma, which validates the choice of $\mathrm{W}$ as a base space.

Lemma 3.2. $\left|\left\{f_{\varphi, \Phi} \mid \varphi \in_{f} \mathcal{B} \wedge \Phi \in_{f} \mathcal{E} \wedge f_{\varphi, \Phi} \cap(p \times D) \neq \emptyset\right\}\right|=O(1)$, for all $p \in \mathrm{W}$.

Proof. The subspace $p \times D$ of the configuration space is intersected by constraint hypersurfaces $f_{\varphi, \Phi}$. A point in $f_{\varphi, \Phi} \cap(p \times D)$ corresponds to a placement of the robot $\mathcal{B}$ in which its reference point is positioned at $p$ and its feature $\varphi$ touches an obstacle feature $\Phi$. This feature $\Phi$ must necessarily belong to one of the $O(1)$ obstacles that can be touched by $\mathcal{B}$ while its reference point is fixed at $p$. Combined with the constant complexity of $\mathcal{B}$ itself, this implies that there exist only a constant number of pairs $(\varphi, \Phi)$ for which $f_{\varphi, \Phi}$ intersects $p \times D$.

We define a partition of the workspace that is subject to constraints that are formulated exclusively in the workspace. The partition subsequently turns out to be a valid base partition for a decomposition of the configuration space into cylinders. The notions of grown obstacles and coverage facilitate the definition of the workspace partition.

Definition 3.3 (Grown Obstacle $G(E, \rho)$ ). Let $E$ be an obstacle in $\mathbb{R}^{d}$ and let $\rho \in \mathbb{R}^{+}$. The $\rho$-grown obstacle $E$ is defined as

$$
G(E, \rho)=\left\{p \in \mathbb{R}^{d} \mid d(p, E) \leq \rho\right\}
$$

Definition 3.4 (Coverage $\operatorname{Cov}(R))$. Let $R \subseteq \mathrm{W}=\mathbb{R}^{d}$.

$$
\operatorname{Cov}(R)=\left\{E \in \mathcal{E} \mid R \cap G\left(E, \rho_{\mathcal{B}}\right) \neq \emptyset\right\} .
$$

Hence, $\operatorname{Cov}(R)$ is the set of obstacles $E$ whose corresponding grown obstacles $G\left(E, \rho_{\mathcal{B}}\right)$ intersect $R$.

It is clear that the robot's reference point must lie inside the grown obstacle $G\left(E, \rho_{\mathcal{B}}\right)$ in order for a feature $\varphi$ of the robot $\mathcal{B}$ to be in contact with a feature $\Phi$ of the obstacle $E$; if the reference point lies outside $G\left(E, \rho_{\mathcal{B}}\right)$ there is no danger for $\varphi \in_{f} \mathcal{B}$ of colliding with $\Phi \in_{f} E$. In other words, a configuration space cylinder $R \times D$ can only intersect a constraint hypersurface $f_{\varphi, \Phi}$ with $\Phi \in_{f} E$ if its projection $R$ intersects $G\left(E, \rho_{\mathcal{B}}\right)$. A formalization of these observations is given by Lemma 3.5; it supplies some kind of simple outer approximation of the location of a constraint hypersurface in configuration space.

Lemma 3.5. $f_{\varphi, \Phi} \subseteq G\left(E, \rho_{\mathcal{B}}\right) \times D$, for all $\varphi \in_{f} \mathcal{B}$ and $\Phi \in_{f} E$. 
A consequence of Lemma 3.5 is that the configuration space cylinder $R \times D$ corresponding to a region $R$ that is intersected by $O(1)$ grown obstacles is itself intersected by at most $O(1)$ constraint hypersurfaces. Lemma 3.6 uses the definition of coverage to obtain a compact statement of the relation between the grown obstacles in the workspace and the constraint hypersurfaces in the configuration space.

Lemma 3.6. Let $R \subseteq \mathrm{W}=\mathbb{R}^{d}$ be such that $|\operatorname{Cov}(R)|=O(1)$. Then

$$
\left|\left\{f_{\varphi, \Phi} \mid \varphi \in_{f} \mathcal{B} \wedge \Phi \in_{f} \mathcal{E} \wedge f_{\varphi, \Phi} \cap(R \times D) \neq \emptyset\right\}\right|=O(1) .
$$

Proof. Take a constraint hypersurface $f_{\varphi, \Phi} \cap(R \times D) \neq \emptyset$. Now let $E$ be such that $\Phi \in_{f} E$. By Lemma 3.5, $f_{\varphi, \Phi} \subseteq G\left(E, \rho_{\mathcal{B}}\right) \times D$. Hence, necessarily $(R \times D) \cap$ $\left(G\left(E, \rho_{\mathcal{B}}\right) \times D\right) \neq \emptyset$ and thus $R \cap G\left(E, \rho_{\mathcal{B}}\right) \neq \emptyset$. By the definition of $\operatorname{Cov}(R)$ and the assumption $|\operatorname{Cov}(R)|=O(1)$, it follows that there are only $O(1)$ obstacles $E$ such that $R \cap G\left(E, \rho_{\mathcal{B}}\right) \neq \emptyset$. Due to the constant complexity of these obstacles and the robot, there is only a constant number of hypersurfaces $f_{\varphi, \Phi}$ with $f_{\varphi, \Phi} \cap(R \times D) \neq \emptyset$.

The lemma states that any region $R$ with $|\operatorname{Cov}(R)|=O(1)$ is guaranteed to satisfy the constraint on the regions of the base partition requiring that the corresponding cylinder is intersected by $O(1)$ constraint hypersurfaces. As a consequence, a decomposition of the workspace $\mathrm{W}$ into constant-complexity regions $R$ with $|\operatorname{Cov}(R)|=O(1)$ is a valid base partition of the base space $B=\mathrm{W}$. We refer to workspace partitions of this kind as cc-partitions (constant-size coverage, constant-complexity).

Definition 3.7 (cc-Partition). A cc-partition $V$ of a workspace $\mathrm{W}$ with obstacles $\mathcal{E}$ is a partition of $\mathrm{W}$ into constant-complexity regions $R$ satisfying $|\operatorname{Cov}(R)|=O(1)$.

The constant-size coverage constraint $|\operatorname{Cov}(R)|=O(1)$ replaces the constraint $\left|\left\{f_{\varphi, \Phi} \mid \varphi \in_{f} \mathcal{B} \wedge \Phi \in_{f} \mathcal{E} \wedge f_{\varphi, \Phi} \cap(R \times D) \neq \emptyset\right\}\right|=O(1)$; the new constraint is simpler because it is truly a constraint in the workspace. The result in Lemma 3.6 and the definition of cc-partitions, however, would be completely useless if a partition of $\mathrm{W}$ into regions $R$ with $|\operatorname{Cov}(R)|=O(1)$ does not exist. Note that the existence of such a partition solely depends on the absence of points $p \in \mathrm{W}$ that are contained in $\omega(1)$ grown obstacles. Fortunately, such points indeed do not exist since the space $\mathbb{R}^{d}$ with the grown obstacles $G\left(E, \rho_{\mathcal{B}}\right)$ is a low obstacle density space. The fact follows immediately from Theorem 2.9, noting that each grown obstacle $G\left(E, \rho_{\mathcal{B}}\right)$ is a $\rho_{\mathcal{B}}$-wrapping and, by $\rho_{\mathcal{B}} \leq b \cdot \rho$, also a $(b \cdot \rho)$-wrapping of the obstacle $E$ itself. As a result, it is indeed possible to partition the low obstacle density workspace $\mathrm{W}$ into regions with constant-size coverage.

Lemma 3.8. A low obstacle density workspace $\mathrm{W}$ can be partitioned into regions $R$ with $|\operatorname{Cov}(R)|=O(1)$.

Notice that, by Theorem 2.4, the arrangement $\mathcal{A}(\partial G)$ of grown obstacle boundaries $\partial G\left(E, \rho_{\mathcal{B}}\right)$ even partitions $\mathrm{W}=\mathbb{R}^{d}$ into $O(n)$ regions $R$ with $|\operatorname{Cov}(R)|=O(1)$, as each $d$-cell of the arrangement is a subset of the intersection of $O(1)$ grown obstacles. 
Unfortunately, it is not a cc-partition, because the $d$-cells may have more than constant complexity. It can though be refined into one; in the case of a planar workspace, the partition is easily refined into a cc-partition of $O(n)$ size by means of a vertical decomposition [31], [33]. The decomposition procedure discussed in Section 4, however, gives a cc-partition of linear size for any dimension.

In summary, we have found that a cc-partition of a low obstacle density workspace always exists. The cc-partition of the workspace corresponds, by Lemma 3.6, to a decomposition of the configuration space into constant-complexity cylinders that are intersected by no more than a constant number of constraint hypersurfaces. As a result, the cc-partition is a valid partition of the base space $\mathrm{W}$ allowing for application of the transformation algorithm from Section 3.1.

The compact algorithm LODMOT given below combines the search for a small ccpartition with its transformation into a cell decomposition of the free space. Besides the cc-partition regions, gathered in a set $V_{\mathrm{W}}$, the first step is to report the adjacencies of the cc-partition regions in a set $E_{\mathrm{W}}$, and the function $\operatorname{Cov}: V_{\mathrm{W}} \rightarrow \mathcal{P}(\mathcal{E})$ mapping each region $R \in V_{\mathrm{W}}$ onto the (constant-cardinality) set of obstacles $E \in \mathcal{E}$ with $G\left(E, \rho_{\mathcal{B}}\right) \cap R \neq \emptyset$. We denote the time required to compute the triple $\left(V_{\mathrm{W}}, E_{\mathrm{W}}, C o v\right)$ by $T(n)$, where the argument $n$ represents the number of obstacles in $\mathcal{E}$.

\section{Algorithm LODMOT}

Find a cc-partition graph $\left(V_{\mathrm{W}}, E_{\mathrm{W}}\right)$ and compute $\operatorname{Cov}: V_{\mathrm{W}} \rightarrow \mathcal{P}(\mathcal{E})$;

$\left(V_{C}, E_{C}\right):=\operatorname{TranSFORM}\left(\left(V_{\mathrm{W}}, E_{\mathrm{W}}\right)\right)$

The $\left|V_{\mathrm{W}}\right|$ precomputed sets $\operatorname{Cov}(R)$ facilitate the constant-time computation of the constraint hypersurface arrangement $\mathcal{A}$ in step 1 of the first for-loop of the transformation algorithm (TRANSFORM). To verify this statement, we refine that step to

$$
\begin{aligned}
& \text { 1.1. } F:=\emptyset ; \\
& \text { 1.2. for all } \varphi \in_{f} \mathcal{B} \wedge \Phi \in_{f} \operatorname{Cov}(R) \text { do } \\
& \text { 1.2.1. compute } f_{\varphi, \Phi} ; \\
& \text { 1.2.2. } F:=F \cup\left\{f_{\varphi, \Phi}\right\} ;
\end{aligned}
$$

1.3. compute the arrangement $\mathcal{A}$ of all $f \in F$;

A closer look at the refinement reveals that $\mathcal{A}$ is now the arrangement of all constraint hypersurfaces in a set $F=\left\{f_{\varphi, \Phi} \mid \varphi \in_{f} \mathcal{B} \wedge \Phi \in_{f} \operatorname{Cov}(R)\right\}$, which is in fact a superset of the set of hypersurfaces $f_{\varphi, \Phi}$ satisfying $f_{\varphi, \Phi} \cap(R \times D) \neq \emptyset$. Fortunately, the easily computable set $F$ contains only a constant number of hypersurfaces, due to the constant cardinality of $\operatorname{Cov}(R)$ and the constant complexity of $\mathcal{B}$ and the individual obstacles $E$. It is clear that the restriction of the arrangement $\mathcal{A}$ to the cylinder $R \times D$ is equivalent to the restriction to $R \times D$ of the arrangement of hypersurfaces $f_{\varphi, \Phi}$ with $f_{\varphi, \Phi} \cap(R \times$ $D) \neq \emptyset$. The techniques by Schwartz and Sharir from [23] may be useful to compute a decomposition of the free part FP $\cap(R \times D)$ of a cylinder $R \times D$.

The refinement of step 1 of the first for-loop verifies the running time of $O\left(\left|V_{\mathrm{W}}\right|\right)$ for the first for-loop of the transformation. The running time of the entire algorithm LODMOT becomes $O\left(\left|V_{\mathrm{W}}\right|+\left|E_{\mathrm{W}}\right|+T(n)\right)$, by Lemma 3.1 and the assumption that the computation of the cc-partition and function $\operatorname{Cov}$ takes $T(n)$ time. The $O\left(\left|V_{\mathrm{W}}\right|+\left|E_{\mathrm{W}}\right|+T(n)\right)$ time bound emphasizes once again that the efficiency of LODMOT is fully determined by 
the size of the partition $\left(V_{\mathrm{W}}, E_{\mathrm{W}}\right)$ and the time to compute it along with Cov: $V_{\mathrm{W}} \rightarrow$ $\mathcal{P}(\mathcal{E})$. Since the time $T(n)$ to compute the graph and the function dominates the time $O\left(\left|V_{\mathrm{W}}\right|+\left|E_{\mathrm{W}}\right|\right)$ to just report both, we may conclude that the $T(n)$-factor dominates the running time of the algorithm LODMOT, which may therefore be said to equal $O(T(n))$.

Theorem 3.9. Let $\mathrm{W}$ with a set $\mathcal{E}$ of obstacles of size at least $\rho$ be a low obstacle density workspace. Let $\mathcal{B}$ be a constant-complexity robot with reach $\rho_{\mathcal{B}} \leq b \cdot \rho$, for some constant $b \geq 0$, moving in $\mathrm{W}$. Furthermore, let $C=\mathrm{W} \times D$ be the configuration space of $\mathcal{B}$. Then the algorithm LODMOT computes the connectivity graph $\left(V_{C}, E_{C}\right)$ with $\left|V_{C}\right|=O\left(\left|V_{\mathrm{W}}\right|\right)$ and $\left|E_{C}\right|=O\left(\left|E_{\mathrm{W}}\right|\right)$ of a cell decomposition of the free space $\mathrm{FP} \subseteq C$ in time $O(T(n))$, where $\left(V_{\mathrm{W}}, E_{\mathrm{W}}\right)$ is a cc-partition of $\mathrm{W}$ and $T(n)$ is the time to compute $\left(V_{\mathrm{W}}, E_{\mathrm{W}}\right)$ along with Cov: $V_{\mathrm{W}} \rightarrow \mathcal{P}(\mathcal{E})$.

Theorem 3.9 reduces the problem of finding a decomposition of certain $q$-cells in an arrangement in the $q$-dimensional configuration space to the problem of finding some constrained partition of the $d$-dimensional workspace $(d \leq q)$.

The incorporation of self-collisions has no major implications for the approach outlined above. The constant number of additional constraint hypersurfaces induced by the self-collisions of the constant-complexity robot does not increase the asymptotic complexity of the arrangement inside any cylinder $R \times D$. Therefore, the combinatorial and algorithmic considerations of this section apply without restrictions. The reader is referred to [31] for further details.

\section{A Linear Size Base Partition}

In Section 3.2 we have reduced the low density motion planning problem for a free-flying robot to the problem of finding a cc-partition of the workspace. A cc-partition subdivides the robot's workspace W into constant-complexity regions that intersect no more than a constant number of grown obstacles. The complexity of this partition, i.e., the number of regions and region adjacencies, and the time to compute it determine the running time of the resulting motion planning algorithm, so we must try to find a small and efficiently computable cc-partition. This section discusses a cc-partition of linear size, which can be computed in near-linear time.

We obtain a cc-partition of the workspace by applying a simplified version of the first stage of de Berg's binary space partition algorithm [4] to the set $\left\{G\left(E, \rho_{\mathcal{B}}\right) \mid E \in \mathcal{E}\right\}$ of grown obstacles. The procedure takes the set of vertices of the axis-parallel bounding boxes of the grown obstacles as input and outputs a decomposition of the workspace into a linear number of hypercubic and L-shaped regions without bounding box vertices in their interiors. It turns out that each of these regions intersects only a constant number of grown obstacles. Below, we explain the procedure in detail.

Let $S$ be a set of $n$ objects and let $\Sigma$ be the set of vertices of the axis-parallel bounding boxes of these objects. The set $\Sigma$ contains $2^{d} n$ vertices. For a region $R$, we denote by $\Sigma(R)$ the subset of $\Sigma$ of bounding box vertices contained in the interior of $R$. Assume that all bounding boxes are enclosed by a large axis-parallel hypercube, which contains all vertices of $\Sigma$. Our variant of the first stage of the binary space partition algorithm 

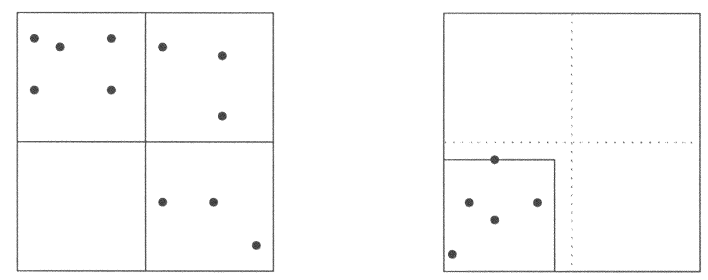

Fig. 5. A useful and useless $2^{d}$-tree split. In the latter case the square is subdivided into an L-shaped region and a square.

by de Berg [4] recursively subdivides this large hypercube until all resulting regions $R$ satisfy $\Sigma(R)=\emptyset$. Let $C$ be a hypercube with $\Sigma(C) \neq \emptyset$ and let $C_{1}, \ldots, C_{2^{d}}$ be the equally sized subhypercubes resulting from a $2^{d}$-tree split of $C$, i.e., from cutting $C$ with the $d$ hyperplanes perpendicular to and bisecting its edges. Note that every subhypercube $C_{i}$ shares exactly one corner with $C$. A $2^{d}$-tree split is called useless if all vertices of $\Sigma(C)$ lie in a single subhypercube $C_{i}$ of $C$, and is called useful otherwise. The hypercube $C$ is subdivided in one of the following ways:

If the $2^{d}$-tree split of $C$ is useful, then perform the $2^{d}$-tree split.

If the $2^{d}$-tree split of $C$ is useless, then replace the single sub-hypercube $C_{i}$ containing all vertices of $\Sigma(C)$ by the smallest hypercube that shares a corner with $C$ and still contains all vertices of $\Sigma(C)$. The resulting hypercube $C^{\prime} \subseteq C$ has one of the vertices of $\Sigma(C)$ on its boundary. Although de Berg refines the complement $C \backslash C^{\prime}$ into $d$ boxes in order to obtain a binary space partition, we simply split $C$ into the smaller hypercube $C^{\prime}$ and the "L-shaped" complement $C \backslash C^{\prime}$, which satisfies $\Sigma\left(C \backslash C^{\prime}\right) \neq \emptyset$. We refer to this type of split of $C$ as an L-split.

Figure 5 gives two-dimensional examples of both types of splits. The recursive splits lead to a decomposition into $O(n)$ hypercubic and L-shaped regions that have no vertices from $\Sigma$ in their interiors. The computation of the tree corresponding to the recursive decomposition-along with the sets of objects intersecting each of the regions-takes $O(n \log n)$ time. The nodes of the tree can be rearranged in $O(n \log n)$ time into a second tree of height $O(\log n)$ (see [4] for details). This second tree allows us to do point location in the decomposition in $O(\log n)$ time, provided that it is possible to determine in constant time for every internal node $v$ whether a query point lies in one of the regions associated with the children of $v$, or in the complement of the region associated with $v$. The condition is true for our decomposition, because its regions are either hypercubes or L-shapes. We use the point location structure during computation of the region adjacencies.

De Berg [4] shows that, under the weak condition of unclutteredness, the number of objects from $S$ intersecting each of the boxes of the decomposition is constant. Since our L-shaped regions are the union of a constant number of such boxes (see above), this property is valid for our decomposition as well. Definition 4.1 recalls the unclutteredness condition. 
Definition 4.1 (Unclutteredness). Let $\mathbb{R}^{d}$ be a space with a set $S$ of objects. The set $S$ satisfies the unclutteredness condition if there is a constant $\kappa$ such that any hypercube whose interior does not contain a vertex of one of the bounding boxes of $S$ is intersected by at most $\kappa$ objects of $S$.

In [5] de Berg et al. study the relations between various realistic assumptions on sets of geometric objects, including fatness, unclutteredness, and low density. Among their results is the following relation.

Lemma 4.2 [5]. Let $\mathbb{R}^{d}$ with a set $S$ of objects satisfy the low object density property. Then $\mathbb{R}^{d}$ with $S$ satisfies the unclutteredness condition.

We now apply the recursive decomposition scheme to the set $\left\{G\left(E, \rho_{\mathcal{B}}\right) \mid E \in \mathcal{E}\right\}$. As all grown obstacles are $(b \cdot \rho)$-wrappings of the original obstacles, and the original obstacles have size at least $\rho$, we know by Theorem 2.9 that the workspace with the set $\left\{G\left(E, \rho_{\mathcal{B}}\right) \mid E \in \mathcal{E}\right\}$ of grown obstacles is still a low density space. Thus, if we apply the decomposition to $\left\{G\left(E, \rho_{\mathcal{B}}\right) \mid E \in \mathcal{E}\right\}$, then Lemma 4.2 assures that we end up with a partition $V_{\mathrm{W}}$ of the workspace into regions $R$ that each intersect at most a constant number of grown obstacles, or, in other words, with $|\operatorname{Cov}(R)|=O(1)$. As the boxes clearly have constant complexity in a fixed dimension $d$, we find that the partition $V_{\mathrm{W}}$ is a cc-partition. The set $V_{\mathrm{W}}$ has size $O(n)$ and is computable, along with the mapping Cov: $V_{\mathrm{W}} \rightarrow \mathcal{P}(\mathcal{E})$, in $O(n \log n)$ time.

It remains to bound the number of pairwise adjacencies of regions of $V_{\mathrm{W}}$, and to show how to compute the set of adjacencies efficiently. It turns out to be convenient (at certain occasions) to cover the L-shaped regions in our decomposition by imaginary hypercubes. In an L-split, a hypercube $C$ is subdivided into a small hypercube $C^{\prime}$ and an L-shaped complement $C \backslash C^{\prime}$. Let $s$ and $s^{\prime}$ be the side lengths of the hypercubes $C$ and $C^{\prime}$, respectively. We cover $C \backslash C^{\prime}$ by $2^{d}-1$ (which is a constant in a fixed dimension $d$ ) imaginary hypercubes of side length $s-s^{\prime}$. This is achieved as follows: for each of the $2^{d}-1$ corners of $C$ not occupied by $C^{\prime}$, we take the imaginary hypercube of side length $s-s^{\prime}$ that lies entirely inside $C$ and has one of its corners coinciding with that corner of $C$. Figure 6 shows the three squares that cover a two-dimensional L-shaped region. Each L-shaped region can thus be covered by $2^{d}-1$ imaginary hypercubes. The $O(n)$ hypercubes from the decomposition and the $O(n)$ imaginary hypercubes jointly cover

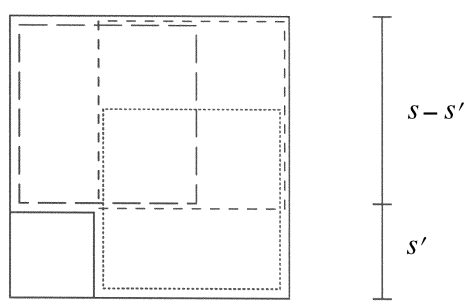

Fig. 6. Three imaginary squares—-shown slightly smaller than their actual size for reasons of clarity—cover the L-shaped region. 
the entire workspace. A useful observation is that if two hypercubes are adjacent, then one of them has a corner in the interior of a facet of the other hypercube, unless both hypercubes are of equal size and share an entire facet. In this specific case, however, the center of one these facets, i.e., the point with equal distance to all facet boundaries, lies in the interior of the other facet (and vice versa). In summary, if we define the set of characteristic points of a hypercube to consist of its $2^{d}$ corners and its $2 d$ facet centers, then for any two adjacent hypercubes, one of them has a characteristic point in the interior of a facet of the other.

Lemma 4.3. The set $E_{\mathrm{W}}$ of pairwise adjacencies of regions in $V_{\mathrm{W}}$ has size $O(n)$.

Proof. If we replace each L-shaped region by the $2^{d}-1$ hypercubes, the number of pairwise adjacencies of the hypercubes in the newly obtained covering of the workspace is larger than the number of pairwise adjacencies of regions of the original decomposition. Hence, we can bound the size of $E_{\mathrm{W}}$ by bounding the number of adjacencies in the covering.

We first show how to charge every pairwise adjacency to a characteristic point on a hypercube and then bound the number of times a point gets charged by a constant. Recall that one of two adjacent hypercubes has a characteristic point in the interior of a facet of the other hypercube. We charge the adjacency to this point. As a region of the decomposition is covered by at most $2^{d}-1$ hypercubes simultaneously, no characterstic point can lie on the boundary of more than $2^{d}-1$ hypercubes. A characteristic point can therefore lie on the common boundary of at most a constant number of pairs of hypercubes. In other words, each of the $O(n)$ characteristic points is involved in no more than a constant number of hypercube adjacencies. This combined with the fact that every adjacency can be charged to a characteristic point yields the desired result.

We compute the set $E_{\mathrm{W}}$ of adjacencies by a reverse execution of the subdivision process: starting from the hypercubic and L-shaped regions of the final decomposition, we repeatedly join the regions resulting from a single split until we obtain the initial hypercube containing all grown obstacles and their bounding boxes. While joining the regions resulting from a split, we compute all adjacencies of regions from $V_{\mathrm{W}}$ that are created by doing so. For each of the $2 d$ facets $f$ of a hypercube $C$ involved in a joining step, we take care to have available the set $\gamma(f)$ of subregions of $C$ (from $V_{\mathrm{W}}$ ) that share a part of their boundary with $f$.

First, let us see what happens if we join an L-shaped region $L$ and a hypercube $C$. Recall that $L$ is a region of the final decomposition $V_{\mathrm{W}}$. The region $L$ becomes adjacent to all regions in $C$ that share a facet with the $d$ facets of $C$ that are "glued" onto facets of $L$ (see Fig. 7 for a three-dimensional example). In other words, for each such facet $f$ of $C$, the region $L$ becomes adjacent to all regions in $\gamma(f)$. The time required to report all different adjacencies of $L$ is clearly proportional to the number of such adjacencies. The sets of regions stored with the facets of the hypercubic union of $L$ and $C$ are easily computed from $L$ and the sets stored with the facets of $C$.

Second, let us see what happens if we join $2^{d}$ equally sized hypercubes into one larger hypercube. The basic task, which has to be performed $d 2^{d-1}$ times, is to glue a $(d-1)$-dimensional facet $f$ of a hypercube $C$ onto a $(d-1)$-dimensional facet $f^{\prime}$ of 


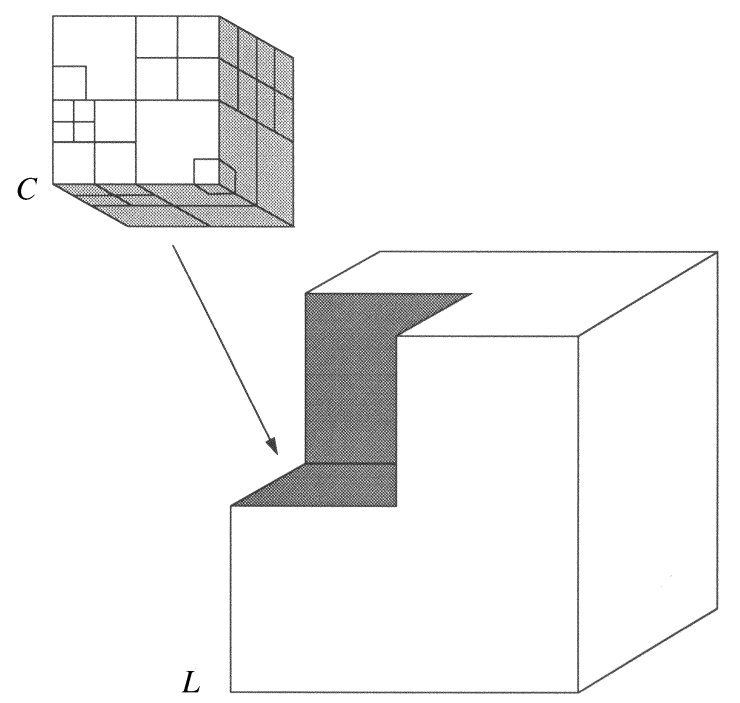

Fig. 7. The grey facets of $C$ are glued onto facets of $L: L$ becomes adjacent to all regions in $C$ that share a part of their boundary with the grey facets.

another hypercube $C^{\prime}$ (see Fig. 8 for a three-dimensional example). This will cause pairs of regions from $C$ and $C^{\prime}$, or, more specifically, pairs of regions from $\gamma(f)$ and $\gamma\left(f^{\prime}\right)$, to become adjacent. To compute the adjacencies, we replace each L-shaped region in $\gamma(f)$ and $\gamma\left(f^{\prime}\right)$ by the $2^{d}-1$ imaginary hypercubes that cover it. Let $m$ and $m^{\prime}$ be the sizes of the sets of hypercubes now sharing a facet with $f$ and $f^{\prime}$, respectively, hence $m=|\gamma(f)|$ and $m^{\prime}=\left|\gamma\left(f^{\prime}\right)\right|$. As each adjacency can be charged to a characteristic point of a hypercube from one of the two sets and no such point gets charged more than a constant number of times (see the proof of Lemma 4.3), the number of adjacencies is

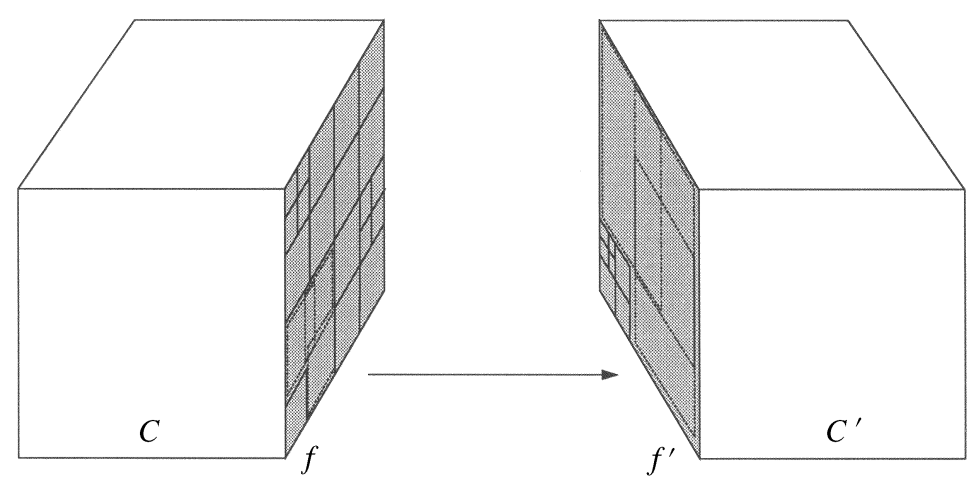

Fig. 8. The grey facets $f$ of $C$ and $f^{\prime}$ of $C^{\prime}$ are glued onto each other. Pairs of regions from $C$ and $C^{\prime}$, or, more specifically, pairs of regions sharing a facet with $f$ and $f^{\prime}$, become adjacent. 
$O\left(m+m^{\prime}\right)$. Because every hypercube from $\gamma(f)$ is adjacent to at least one hypercube from $\gamma\left(f^{\prime}\right)$, we can even conclude that the number of adjacencies is $\Theta\left(m+m^{\prime}\right)$.

Using the observation that one of two adjacent hypercubes has a characteristic point in the interior of a facet of the other, we find the following simple strategy for computing all adjacencies: determine for each characteristic point of a hypercube in $\gamma(f)$ the hypercube in $\gamma\left(f^{\prime}\right)$ containing it, and vice versa. Note that we can restrict ourselves to querying with the characteristic points on the joined facets $f$ and $f^{\prime}$. Keeping in mind that our ultimate aim is to compute the pairwise adjacencies of regions from $V_{\mathrm{W}}$ we can just as well replace this strategy by: determine for each characteristic point of a hypercube in $\gamma(f)$ the region (in $C^{\prime}$ ) containing it, and vice versa. The point location structure of the decomposition allows us to find the region containing a query point in $O(\log n)$ time. We observe that all our query points lie on the common boundary of at least two regions. To overcome this complication, we (symbolically) move the query points from $\gamma(f)$ perpendicularly away from $f$ (and $C$ ), thereby moving a query point in the interior of a facet of an adjacent region into the interior of that region. The queries with the $O(m)$ characteristic points of the hypercubes in $\gamma(f)$ can be solved in $O(m \log n)$ time. Likewise, we can solve the reverse problem in $O\left(m^{\prime} \log n\right)$ time. No adjacency will be reported more than a constant number of times.

The preceding analysis shows that the time required to compute the $k$ adjacencies created by uniting $C$ and $C^{\prime}$ is bounded by $O(k \log n)$. Moreover, the computation of all $K$ adjacencies resulting from joining the $2^{d}$ equally sized hypercubes into one larger hypercube takes $O(K \log n)$ time. The sets $\gamma(f)$ for each of the facets $f$ of the newly obtained large hypersphere are trivially computable from the appropriate sets stored with the facets of the $2^{d}$ subhypercubes. Combining the time bounds for both types of joining steps and the $O(n)$ bound on the size of $E_{\mathrm{W}}$ given by Lemma 4.3 leads to the following result.

Lemma 4.4. The set $E_{\mathrm{W}}$ of pairwise adjacencies of regions in $V_{\mathrm{W}}$ is computable in $O(n \log n)$ time.

We have found linear-size cc-partitions for workspaces of arbitrary dimension. These partitions are computable in $O(n \log n)$ time. Substitution of these computations into the first step of algorithm LODMOT yields the following result by Theorem 3.9.

Theorem 4.5. The low density motion planning problem for a free-flying robot can be solved in $O(n \log n)$ time.

\section{Conclusion}

We have studied the motion planning problem for a constant-complexity robot $\mathcal{B}$ with $q$ degrees of freedom in a low obstacle density workspace with $n$ constant-complexity obstacles $E \subseteq \mathbb{R}^{d}$, for some constants $d, q \geq 0$. The reach $\rho_{\mathcal{B}}$ of the robot $\mathcal{B}$ is assumed to be bounded from above by $b \cdot \rho$, where $b \geq 0$ is a constant and $\rho$ is a lower bound on the size of any obstacle $E$. The mild assumptions provide a realistic framework for many practical motion planning problems. The complexity of the free space for problems that 
satisfy the assumptions was proven to be $O(n)$ [32], whereas the complexity can easily be as high as $\Omega\left(n^{q}\right)$ when both assumptions are dropped.

Besides having a low combinatorial complexity, the free space for a motion planning problem that fits in our framework also has a beneficial structure. The structure allows for a decomposition of the configuration space $C=\mathrm{W} \times D$ into cylinders, with bases in the workspace $\mathrm{W}$ such that the free space part of every cylinder has constant-complexity. This reduces the problem of finding a cell decomposition of the free space to the problem of finding some constrained partition of the lower-dimensional workspace. A uniform sequence of operations then suffices to transform the workspace partition into a cell decomposition of the free space of asymptotically equal size. The running time of the entire paradigm is determined by the time to compute the workspace partition.

We have shown that optimal $O(n)$ size workspace partitions exist in any fixed dimension $d$. The partitions are computable in $O(n \log n)$ time. These results lead to $O(n \log n)$ algorithms for solving the low density motion planning problem. The bounds show that our approach of decomposing some lower-dimensional subspace of the configuration space (subject to some constraints) results in efficient solutions to the low density motion planning problem.

It is interesting to see if the paradigm for motion planning in environments with low obstacle density applies to other classes of motion planning problems. The general idea of subdividing the configuration space into cylinders-with bases in some lower-dimensional subspace - in which the free space has constant complexity may be applicable to configuration spaces other than $C=\mathrm{W} \times D$. The results from this paper are, for example, immediately applicable to a robot moving in a three-dimensional world while its motion is confined to a plane, e.g., a factory floor [31]. Other possible extensions include motion planning with moving obstacles, multiple robots, and anchored robot arms.

The dynamic version of the low density motion planning problem has been studied by Berretty et al. [6]. They consider a setting in which the obstacles in the workspace move at constant speed along polyline paths. The workspace is assumed to satisfy the low density property at any time. The authors use the ideas from Section 3.1 to find a nearly optimal $O\left(n^{2} \alpha(n) \log ^{3} n\right)$ solution to the problem.

The usual approach to the exact solution of a motion planning problem with $c$ boundedsize robots with configuration spaces $C_{1}, \ldots, C_{c}$ of dimensions $q_{1}, \ldots, q_{c}$ is to regard these robots as one multibody robot. Planning the motion of the multibody robot takes place in the composite configuration space $C=C_{1} \times \cdots \times C_{c}$. We believe the complexity of the free part of $C$ to be close to the realizable lower bound of $\Omega\left(n^{c}\right)$ rather than to the trivial upper bound of $O\left(n^{q}\right)$, with $q=q_{1}+\cdots+q_{c}$. The idea of a decomposition of the free space by means of cylinders seems applicable if the workspace $\mathrm{W}$ is a lowerdimensional subspace of each of the spaces $C_{i}$; in that case, $\mathrm{W}^{c}$ is a valid base space. Vleugels [34] has shown how to restrict the search for a free path for two robots in their composite configuration space $C$ to a collection of lower-dimensional subspaces of $C$, such that a path exists in the subspaces whenever one exists in $C$. The approach leads to $O(n \log n)$ and $O\left(n \log ^{2} n\right)$ algorithms for two robots in two- and higher-dimensional workspaces respectively, even though the complexity of the free spaces can be $\Omega\left(n^{2}\right)$.

For most industrial robot arms, the links close to the hand-the minor axes-are considerably shorter than the links close to the base-the major axes. Consider a $q$-link 
robot arm of which the $m$ minor axes are not too large compared with the obstacles. Let $C$ be the configuration space and assume that $C^{\prime}$ is the $(q-m)$-dimensional subspace corresponding to the major axes. A point $p \in C^{\prime}$ fixes the placements of all major axes. If $m$ is a constant, then the $m$ minor axes can only touch a constant number of obstacles while the major axes are fixed, due to the low obstacle density. As a result, the lifting of the point $p \in C^{\prime}$ into $C$ will be intersected by only a constant number of constraint hypersurfaces, making $C$ a cylindrifiable configuration space and $C^{\prime}$ a valid base space. It is however unclear at the moment how to compute base partitions in $C^{\prime}$.

\section{References}

1. P.K. Agarwal, M.J. Katz, and M. Sharir, Computing depth orders for fat objects and related problems, Computational Geometry: Theory and Applications 5 (1995), 187-206.

2. H. Alt, R. Fleischer, M. Kaufmann, K. Mehlhorn, S. Näher, S. Schirra, and C. Uhrig, Approximate motion planning and the complexity of the boundary of the union of simple geometric figures, Algorithmica $\mathbf{8}$ (1992), 391-406.

3. F. Avnaim, J.-D. Boissonnat, and B. Faverjon, A practical exact motion planning algorithm for polygonal objects amidst polygonal obstacles, Proc. Geometry and Robotics Workshop (J.-D. Boissonnat and J.-P. Laumond, eds.), Lecture Notes in Computer Science, vol. 391, Springer-Verlag, Berlin (1988), pp. 67-86.

4. M. de Berg, Linear size binary space partitions for fat objects, Technical Report UU-CS-1998-12, Dept. of Computer Science, Utrecht University (1998).

5. M. de Berg, M. Katz, A.F. van der Stappen, and J. Vleugels, Realistic input models for geometric algorithms, Proc. 13th Ann. ACM Symp. on Computational Geometry (1997), pp. 294-303.

6. R.-P. Berretty, M. Overmars, and A.F. van der Stappen, Dynamic motion planning in low obstacle density environments, Proc. Workshop on Algorithms and Data Structures (WADS '97), Lecture Notes in Computer Science, vol. 1272, Springer-Verlag, Berlin (1997), pp. 3-16.

7. J.F. Canny, The Complexity of Robot Motion Planning, MIT Press, Cambridge, MA (1988).

8. A. Efrat, G. Rote, and M. Sharir, On the union of fat wedges and separating a collection of segments by a line, Computational Geometry: Theory and Applications 3 (1993), 277-288.

9. D. Halperin and M.H. Overmars, Spheres, molecules, and hidden surface removal, Proc. 10th Ann. ACM Symp. on Computational Geometry (1994), 113-122.

10. M.J. Katz, M.H. Overmars, and M. Sharir, Efficient hidden surface removal for objects with small union size, Computational Geometry: Theory and Applications 2 (1992), 223-234.

11. Y. Ke and J. O'Rourke, Moving a ladder in three dimensions: upper and lower bounds, Proc. 3rd Ann. ACM Symp. on Computational Geometry (1987), pp. 136-145.

12. M. van Kreveld, On fat partitioning, fat covering and the union size of polygons, Computational Geometry: Theory and Applications 9 (1998), 197-210.

13. D. Leven and M. Sharir, Planning a purely translational motion for a convex object in two-dimensional space using generalized Voronoi diagrams, Discrete \& Computational Geometry 2 (1987), 9-31.

14. D. Leven and M. Sharir, On the number of critical free contacts of a convex polygonal object moving in two-dimensional space, Discrete \& Computational Geometry 2 (1987), 255-270.

15. D. Leven and M. Sharir, An efficient and simple motion planning algorithm for a ladder amidst polygonal barriers, Journal of Algorithms 8 (1987), 192-215.

16. J. Matoušek, J. Pach, M. Sharir, S. Sifrony, and E. Welzl, Fat triangles determine linearly many holes, SIAM Journal on Computing 23 (1994), 154-169.

17. C. Ó’Dúnlaing, M. Sharir, and C.-K. Yap, Retraction: A new approach to motion planning, Proc. 15 th Ann. ACM Symp. on the Theory of Computing (1983), pp. 207-220.

18. C. Ó'Dúnlaing and C.-K. Yap, A retraction method for planning the motion of a disc, Journal of Algorithms 6 (1985), 104-111.

19. M.H. Overmars, Point location in fat subdivisions, Information Processing Letters 44 (1992), 261-265.

20. M.H. Overmars and A.F. van der Stappen, Range searching and point location among fat objects, Journal of Algorithms 21 (1996), 629-656. 
21. P. Pignon, Structuration de l' espace pour une planification hiérarchisée des trajectoires de robots mobiles, Ph.D. Thesis, LAAS-CNRS and Université Paul Sabatier de Toulouse, Rapport LAAS No. 93395 (1993) (in French).

22. J.T. Schwartz and M. Sharir, On the piano movers' problem: I. The case of a two-dimensional rigid polygonal body moving amidst polygonal boundaries, Communications on Pure and Applied Mathematics 36 (1983), 345-398.

23. J.T. Schwartz and M. Sharir, On the piano movers' problem: II. General techniques for computing topological properties of real algebraic manifolds, Advances in Applied Mathematics 4 (1983), 298-351.

24. J.T. Schwartz and M. Sharir, On the piano movers' problem: III. Coordinating the motion of several independent bodies: the special case of circular bodies moving amidst polygonal barriers, International Journal of Robotics Research 2 (1983), 46-75.

25. J.T. Schwartz and M. Sharir, On the piano movers' problem: V. The case of a rod moving in threedimensional space amidst polyhedral obstacles, Communications on Pure and Applied Mathematics 37 (1984), 815-848.

26. J.T. Schwartz and M. Sharir, Efficient motion planning algorithms in environments of bounded local complexity, Report 164, Department of Computer Science, Courant Inst. Math. Sci., New York, NY (1985).

27. M. Sharir, Efficient algorithms for planning purely translational collision-free motion in two and three dimensions, Proc. IEEE Internat. Conf. on Robotics and Automation, Raleigh, NC (1987), pp. 1326-1331.

28. M. Sharir and E. Ariel-Sheffi, On the piano movers' problem: IV. Various decomposable two-dimensional motion planning problems, Communications on Pure and Applied Mathematics 37 (1984), 479-493.

29. S. Sifrony and M. Sharir, A new efficient motion planning algorithm for a rod in two-dimensional polygonal space, Algorithmica 2 (1987), 367-402.

30. A.F. van der Stappen, The complexity of the free space for motion planning amidst fat obstacles, Journal of Intelligent and Robotic Systems 11 (1994), 21-44.

31. A.F. van der Stappen, Motion planning amidst fat obstacles, Ph.D. Thesis, Dept. of Computer Science, Utrecht University (1994).

32. A.F. van der Stappen, D. Halperin, and M.H. Overmars, The complexity of the free space for a robot moving amidst fat obstacles, Computational Geometry: Theory and Applications 3 (1993), 353-373.

33. A.F. van der Stappen and M.H. Overmars, Motion planning amidst fat obstacles, Proc. 10th Ann. ACM Symp. on Computational Geometry (1994), pp. 31-40.

34. J. Vleugels, On fatness and fitness-realistic input models for geometric algorithms, Ph.D. Thesis, Dept. of Computer Science, Utrecht University (1997).

Received October 17, 1995, and in revised form July 8, 1997, and February 23, 1998. 